\title{
Milchproduktion in Deutschland und Europa nach der Liberalisierung - Abschätzung künftiger Entwicklungen mit unterschiedlichen analytischen Ansätzen
}

\author{
Dissertation \\ zur Erlangung des Doktorgrades \\ der Fakultät für Agrarwissenschaften \\ der Georg-August-Universität Göttingen
}

vorgelegt von

Birthe Johanna Lassen

geboren in Frankfurt am Main

Göttingen, im September 2011 
D 7

1. Referent: Prof. Dr. Folkhard Isermeyer

2. Korreferent: Prof. Dr. Ludwig Theuvsen

Tag der mündlichen Prüfung: 07.11.2011 


\section{Danksagung}

Ziemlich genau fünf Jahre sind seit dem ersten Tag als Doktorandin vergangen. Jahre, in denen ich viel gelernt und gesehen habe, aber auch Jahre, in denen ich so manches Mal dachte, dass ich nie fertig werde.

Dafür, dass ich nun die fertige Arbeit in den Händen halten darf, gebührt vielen Menschen ein großer Dank. Dieser gilt in erster Linie meinem Doktorvater Professor Folkhard Isermeyer, von dem ich in der Vergangenheit sehr viel lernen durfte. Er hat mir beigebracht, im Raum stehende Hypothesen zu hinterfragen, ihre theoretische Basis zu prüfen und den eigenen Analysen den ihnen gebührenden Wert beizumessen. Bei Professor Ludwig Theuvsen bedanke ich mich sehr herzlich für die Übernahme des Korreferates und für die stets motivierende Unterstützung.

Befragungen, wie ich sie in den letzten Jahren durchgeführt habe, erfordern viele helfende Hände. Mein Dank gilt deshalb unseren internationalen Projektpartnern, den EDFSTARS, und unseren deutschen Betriebsberatern im Netzwerk. Darüber hinaus bedanke ich mich bei Herrn Dr. Rüther (Landvolk Niedersachsen), Herrn Holtorf (frischli), Herrn Dr. Storm (DKB), Herrn Blarr (AVS) und Herrn Mirbach (DLG Spitzenbetriebe) für die langjährige Unterstützung. Mein ganz besonderer Dank gilt jedoch den teilnehmenden Milcherzeugern, ohne deren alljährlichen Einsatz die Analysen in der vorliegenden Form nicht möglich gewesen wären. Unsere ,guten Geister“ im Institut für Betriebswirtschaft waren eine unerlässliche Hilfe beim Eingeben der Fragebögen und in allen Formatierungsfragen. Auch Michael Wolter und Rhena Kröger waren eine große Hilfe. Zazie von Davier erklärte mir in letzter Minute die Tücken von SPSS, dafür bin ich ihr sehr dankbar. Darüber hinaus bedanke ich mich bei den Kollegen im Institut für Betriebswirtschaft, die mir in den letzten Jahren mit ihrer fachlichen Expertise und ihrem Erfahrungsschatz mit Rat und Tat zur Seite standen.

Insbesondere während der letzten Monate hat mir das Milch-Team im vTI viele zeitliche Freiräume ermöglicht. Vor allem Steffi Wille-Sonk sei an dieser Stelle mehr als herzlich gedankt! In Friederike Schierholz fand ich eine wertvolle, wenn auch leider nur kurzzeitige Kollegin und eine geduldige „Feedbackinstanz“, in Heiko Hansen einen stetigen Motivationsgeber. Das alles wäre ohne die Unterstützung der Institutsleitung nicht möglich gewesen, dafür gilt Hiltrud Nieberg mein Dank. Moralische Unterstützung gab es stets „unterm Dach“ - Klaus Nehring und Daniel Brüggemann zeigten, dass der Einsatz sich lohnt und Janina Krug konnte mit ihrer rheinischen Frohnatur und „Sonne im Herzen“ über manch trüben Moment hinweg trösten.

Auch außerhalb des Institutes fand sich stets ein Resonanzboden für erste Forschungsergebnisse und spannende Diskussionen. Dafür danke ich Denise Völker, Kerstin Reiners und natürlich Arndt Reil, der mich damals eingearbeitet hat und mir seither ein treuer Be- 
gleiter und Diskussionspartner ist. Auch mit Christian Schaper verband mich über die Göttinger Milch-AG hinaus ein steter Austausch.

Bei allem freundschaftlichen, fachlichen und organisatorischen Support in „Agrarkreisen“ wäre diese Doktorarbeit jedoch nie fertig geworden, hätte ich nicht auf den Rückhalt meiner Freunde und Familie bauen können: Andrea und Oliver übernahmen viele EmilDienste, meine Göttinger Mädels, Kerstin und Aniko hatten stets ein offenes Ohr und aufmunternde Worte parat und Friedrich fand die richtigen Worte zum richtigen Zeitpunkt. Bernd gab mir die Ruhe, die Kraft und die Motivation für den Endspurt.

Last but not least haben meine Eltern und Geschwister stets an mich geglaubt und daran, dass es neben meiner Großmutter eines Tages noch eine zweite Frau Dr. Lassen in der Familie geben wird. Dafür danke ich ihnen von ganzem Herzen.

Birthe Johanna Lassen 


\section{Inhaltsverzeichnis}

Danksagung $\quad$ i

1 Einleitung 1

2 Regionale Entwicklung der Milchproduktion in Deutschland und Europa 5

Regionaler Strukturwandel in der deutschen Milchproduktion 6

Änderung der Quotenhandelsgebiete in Deutschland - wohin wandert die 27

Milchproduktion

Wettbewerbsfähigkeit der Milchproduktion in Regionen Deutschlands

und Europas

Zukünftige Entwicklung der Milchproduktion in Niedersachsen 66

Produktionskosten und Zukunftseinschätzungen europäischer Milchviehhalter 89

im Vergleich

Milchproduktion in Deutschland: Situation, regionale Trends und Perspektiven 105

Milk Production in the EU

Exkurs: Grünlandnutzung in der EU

Impact of economic and political drivers on grassland use in the EU

\section{Entwicklungen in der Melktechnik}

Zusammenhang zwischen Betriebsstruktur, Melktechnik und Produktivität

- Ergebnisse einer europaweiten Befragung von Milcherzeugern

Trends in der Melktechnik - Ergebnisse einer EU-weiten Umfrage

4 Risikomanagement in Milchviehbetrieben

Risikomanagement in Milchviehbetrieben: Eine empirische Analyse vor dem Hintergrund der sich ändernden EU-Milchmarktpolitik

Risk Management on Dairy Farms: An Empirical Analysis

Risikowahrnehmung in Milchviehbetrieben: Eine empirische Studie zur vergleichenden Bewertung von Politik,- Markt- und Produktionsrisiken

Risk Management in Milk Production: A Study in Five European Countries 


\section{$1 \quad$ Einleitung}




\section{Problemstellung}

Der europäische Milchmarkt wurde in der Vergangenheit stark durch agrarpolitische Maßnahmen reglementiert. Die zunehmende Liberalisierung der Gemeinsamen Agrarpolitik führt jedoch dazu, dass sich sowohl Milcherzeuger als auch verarbeitende Unternehmen neu im überregionalen und internationalen Wettbewerb orientieren müssen. Insbesondere das Ende der Milchquotenregelung im Jahr 2015 führt zu einer neuen Wettbewerbssituation. Während bisher die Produktionsmengen einzelner Mitgliedsstaaten der EU durch die Milchquote festgelegt waren, entfallen diese Produktionsbeschränkungen mit dem Auslaufen der Milchquotenregelung. Dadurch kann es sowohl innerhalb der Länder, als auch zwischen den Mitgliedsstaaten und auf dem Weltmarkt zu Verschiebungen von Marktanteilen kommen. In Deutschland wurden in Vorbereitung auf das Ende der Milchquotenregelung die 21 kleinräumigen Quotenhandelsregionen bereits im Jahr $2007 \mathrm{zu}$ zwei neuen Quotengebieten zusammengefasst (MILCHQUOTV, 2008), so dass bereits erste regionale Wanderungen der Milchquoten zu beobachten sind.

Bisherige Analysen zur Frage der künftigen Entwicklung der regionalen Wettbewerbsfähigkeit kommen aufgrund unterschiedlicher methodischer Ansätze jedoch zu unterschiedlichen Ergebnissen: Während aus Trendfortschreibungen bisheriger Wanderungen der Milchproduktion in Deutschland beispielsweise eher die Einschätzung gewonnen wurde, dass die Milchproduktion künftig weiter auf Grünlandstandorte wandert (GöMANN et al., 2006), zeigen Produktionskostenanalysen Vorteile großbetrieblicher Strukturen in den neuen Bundesländern, so dass hier trotz niedriger Grünlandanteile ebenfalls eine wettbewerbsfähige Milchproduktion erhalten bleiben könnte (ISERMEYER et al., 2006; 2007).

Oft hängen die erzielten Einschätzungen auch von der gewählten Datengrundlage ab. Bei Produktionskostenanalysen müssen sich Wissenschaftler beispielsweise entscheiden: entweder für die Analyse repräsentativer Betriebsdaten, z. B. auf Basis des Testbetriebsnetzes FADN (dieses verfügt jedoch nicht über detaillierte Betriebszweigauswertungen sondern nur über gesamtbetriebliche Daten) oder für detaillierte Betriebszweiganalysen (z. B. von IFCN oder EDF). Diese liegen jedoch nicht für eine repräsentative Betriebsauswahl vor. Diese unterschiedlichen Datensätze führen zu abweichenden Ergebnissen. So ordnen beispielsweise Analysen auf Basis von FADN und IFCN Daten die Wettbewerbsfähigkeit der deutschen Milchproduktion eher im unteren Mittelfeld ein, während EDF-Analysen die Wettbewerbsfähigkeit der deutschen Milchproduktion vergleichsweise hoch einschätzen (LUTTER, 2009).

Komplexe, internationale Agrarsektormodelle (z. B. EDIM, AGMEMOD) kommen wiederum zu anderen Einschätzungen. Während Produktionskostenanalysen die Milchproduktion im Vereinigten Königreich beispielsweise als sehr wettbewerbsfähig einschätzen, zeigen die Analysen der Agrarsektormodelle eher eine niedrige Wettbewerbsfähigkeit für die dortige Milchproduktion (LUTTER, 2009). 
Diese konträren Ergebnisse zeigen, dass die Anwendung nur eines einzelnen methodischen Ansatzes nicht ausreichend ist, wenn komplexe Fragen zur Wettbewerbsfähigkeit von Regionen vor dem Hintergrund aktueller politischer Veränderungen beantwortet werden sollen. Um zu einer abgewogenen Gesamteinschätzung zu gelangen, sollte vielmehr die gleichzeitige Anwendung unterschiedlicher Ansätze erwogen werden.

Neben dem Auslaufen der Milchquotenregelung werden im Zuge der Liberalisierung zudem staatliche Sicherheitsnetze (z. B. Intervention, Exporterstattungen) reduziert und es ist davon auszugehen, dass im Rahmen künftiger WTO-Verhandlungen der Außenschutz für Milchprodukte weiter reduziert wird (MILLER, 2005). Damit spiegeln sich Entwicklungen auf den internationalen Milchmärkten zunehmend in europäischen und deutschen Milchpreisen wider. Dies erhöht für Milcherzeuger die Preis- und Liquiditätsrisiken (SCHAPER et al., 2008).

Die Frage, wie Milcherzeuger künftig strategisch mit zunehmenden Preis- und Liquiditätsrisiken umgehen werden, wurde bisher kaum untersucht. Zwar zeigen vereinzelte Studien, dass Milcherzeuger Markt- und Politikrisiken überwiegend mit Betriebsgrößenwachstum begegnen (KÖHNE, 2000). Internationale Analysen, die sich auf die Milchviehhaltung konzentrieren und zeigen, wie Milcherzeuger die neuen Risiken wahrnehmen und welche strategischen Anpassungsmaßnahmen sie vornehmen, liegen jedoch kaum vor (HoLLMANN-HESPOS, 2003; SCHAPER et al., 2008).

Aus diesen neuen Rahmenbedingungen resultieren zwei unterschiedliche Forschungsfragen:

(1) Welche regionalen Entwicklungen sind in der Milchproduktion national und international künftig zu erwarten?

(2) Wie passen sich Milcherzeuger strategisch und produktionstechnisch an neue Herausforderungen an?

Die Beantwortung dieser Forschungsfragen ist mit den bisherigen Forschungsansätzen auf Grund der teilweise widersprüchlichen Ergebnisse schwierig. Es erscheint deshalb sinnvoll, zusätzlich Unternehmer im Milchmarkt zu befragen. Internationale Befragungskonzepte für Milcherzeuger gibt es bisher jedoch nicht und eine einmalige Befragung von Milcherzeugern ist auf Grund der Komplexität beider Forschungsfragen nicht ausreichend. Es ist deshalb notwendig, ein Konzept zu entwickeln, dass langfristig den Zugang zu aktiven Milcherzeugern ermöglicht, die dann wiederholt befragt werden können. Dabei wäre es wünschenswert, auf eine möglichst gleichbleibende Stichprobe zurückgreifen zu können. 


\section{Zielstellung}

Vor diesem Hintergrund ist es das Ziel der vorliegenden Dissertation,

(1) gleichzeitig unterschiedliche methodische Ansätze anzuwenden auf die Frage der künftigen regionalen Entwicklung der Milchproduktion.

(2) einen empirischen Befragungsansatz zu entwickeln, an dem sich international viele Milcherzeuger freiwillig beteiligen und der so Analysen zu Zusammenhängen zwischen Betriebs- und Standortfaktoren, ökonomischen Parametern, strategischen Fragestellungen oder produktionstechnischen Kennzahlen ermöglicht.

\section{Vorgehensweise}

Entsprechend der unterschiedlichen Forschungsfragen gliedert sich die vorliegende Dissertation in drei Kapitel, in denen im Folgenden die einzelnen, bereits veröffentlichten oder zur Veröffentlichung eingereichten Beiträge vorgestellt werden.

Kapitel 2 widmet sich der Frage der künftigen regionalen Wanderung der Milchproduktion in Deutschland und Europa. Dabei werden zunächst nacheinander drei unterschiedliche methodische Ansätze (Marktanteilsanalyse, Produktionskostenanalyse, Standortfaktorenanalyse) angewandt und zu einem abgewogenen Gesamtbild zusammengeführt. Darüber hinaus werden der entwickelte Befragungsansatz angewendet und Expertengespräche geführt, so dass die drei etablierten Analyseverfahren durch die Einschätzung praktischer Milcherzeuger ergänzt werden.

Weitere Einsatzmöglichkeiten des entwickelten Befragungsansatzes werden in Kapitel 3 dargestellt. Der entwickelte Befragungsansatz wird hier für Analysen zu produktionstechnischen Entwicklungen und deren Auswirkungen auf die Produktivität der Milchvieh haltenden Betriebe eingesetzt. Beispielhaft werden die künftigen Entwicklungen in der Melktechnik analysiert, da der Melkprozess für eine wettbewerbsfähige Milchproduktion von besonderer Bedeutung ist.

In Kapitel 4 werden die Fragen der Risikowahrnehmung und des Risikomanagements von Milchviehhaltern in Deutschland und Europa vor dem Hintergrund der Liberalisierung der Milchmärkte adressiert. Die vorliegenden Studien basieren auf Fallstudien sowie Expertengesprächen in fünf europäischen Ländern.

Eine abschließende Zusammenfassung erfolgt in Kapitel 5. 
2 Regionale Entwicklung der Milchproduktion in Deutschland und Europa 


\section{Regionaler Strukturwandel in der deutschen Milchproduktion}

Birthe Lassen, Folkhard Isermeyer, Carina Friedrich

Dieser Beitrag ist in der Agrarwirtschaft, Band 58, Heft 5/6 auf den Seiten 238 bis 247 erschienen. 


\section{Änderung der Quotenhandelsgebiete in Deutschland - wohin wandert die Milchproduktion?}

Birthe Lassen, Folkhard Isermeyer, Carina Friedrich

Dieser Beitrag wurde auf den KTBL-Tagen am 19. März 2009 zum Thema „Landwirtschaft im Umbruch-Herausforderungen und Lösungen“ in Goslar vorgestellt und ist im Tagungsband des KTBL (KTBL Schrift 474), Seite 144 bis 155 erschienen. 


\section{Wettbewerbsfähigkeit der Milchproduktion in Regionen Deutschlands und Europas}

Birthe Lassen, Folkhard Isermeyer

Dieser Beitrag wurde auf dem X. Brandenburger Nutztierforum "Wie wettbewerbsfähig ist die deutsche Tierhaltung” am 14. Oktober 2009 in Götz vorgestellt und ist der DGfZ-Schriftenreihe Heft 54, Seite 17 bis 39 erschienen. 


\section{Zukünftige Entwicklung der Milchproduktion in Niedersachsen}

Birthe Lassen, Friederike Schierholz, Dr. Jürn Sanders

Dieser Beitrag ist im Yearbook of Socioeconomics in Agriculture, 2011, auf den Seiten 217 bis 243 erschienen. 


\section{Produktionskosten und Zukunftseinschätzungen europäischer Milchviehhalter im Vergleich}

Birthe Lassen, Folkhard Isermeyer, EDF-STAR

Dieser Beitrag ist im Tagungsband zur 2. Göttinger Fachtagung für Milchwirtschaft (Agrarökonomie, Band 3) Seite 5 bis 22 erschienen. 


\section{Milchproduktion in Deutschland: Situation, regionale Trends und Perspektiven}

Dieter Künstling, Birthe Lassen, Bernd Lührmann, Ralf Over

Dieser Beitrag ist im Trendreport Spitzenbetriebe 2010 mit dem Titel „Milchviehhaltung, Erfolgsfaktoren Spitzenbetriebe " auf den Seiten 21 bis 37 erschienen. 


\section{Milk Production in the EU}

Birthe Lassen, Steffi Wille, Oliver von Ledebur

Dieser Beitrag ist im Sammelband „Comércio International de Lácteos“ auf den Seiten 253 bis 272 erschienen. 
Exkurs: Grünlandnutzung in der EU

\section{Impact of economic and political drivers on grassland use in the $\mathbf{E U}$}

Bernhard Osterburg, Folkhard Isermeyer, Birthe Lassen und Norbert Röder

Dieser Beitrag wurde auf der EGF Tagung 2010 zum Thema „Grassland in a changing world - meeting the requirements of producers, livestock, society and the environment" am 30. August 2010 in Kiel vorgestellt und ist in „Grassland in a changing world“, Tagungsband der 23. Konferenz der European Grassland Federation, Seite 14-28, erschienen. 
3 Entwicklungen in der Melktechnik 
Zusammenhang zwischen Betriebsstruktur, Melktechnik und Produktivität - Ergebnisse einer europaweiten Befragung von Milcherzeugern

Birthe Lassen

Dieser Beitrag ist in der Schriftenreihe „Berichte über Landwirtschaft, Zeitschrift für Agrarpolitik und Landwirtschaft, Band 89 (3), Dezember 2011, auf den Seiten 376 bis 399 erschienen. 


\section{Trends in der Melktechnik \\ - Ergebnisse einer EU-weiten Umfrage}

Birthe Lassen

Dieser Beitrag wird auf der 12. Jahrestagung der Wissenschaftlichen Gesellschaft der Milcherzeugerberater (WGM e.V.) am 4. und 5. Oktober 2011 in Aulendorf vorgestell und wird im Tagungsband erscheinen. 
4 Risikomanagement in Milchviehbetrieben 


\section{Risikomanagement in Milchviehbetrieben: Eine empirische Analyse vor dem Hintergrund der sich ändern- den EU-Milchmarktpolitik}

Christian Schaper, Christian Wocken, Klaus Abeln, Birthe Lassen, Sven Schierenbeck, Achim Spiller und Ludwig Theuvsen

Dieser Beitrag wurde auf dem Symposium der Edmund Rehwinkel-Stiftung am 11. April 2008 zu dem Generalthema „Anforderungen an das Risikomanagement in der Landwirtschaft" in Frankfurt am Main vorgestellt und ist in der Schriftenreihe der Landwirtschaftlichen Rentenbank zum Thema „Risikomanagement in der Landwirtschaft“, Band 23, S. 135-184 erschienen. 


\section{Risk Management on Dairy Farms: An Empirical Analysis}

Birthe Lassen, Christian Schaper, Achim Spiller, Ludwig Theuvsen und Christian Wocken

Dieser Beitrag wurde auf der 8th International Conference on Management in AgriFood Chains and Networks vom 29. bis 30. Mai 2008 zum Generalthema „Leadership, Innovation, and Entrepreneurship in Chains and Networks" in den Niederlanden vorgestellt und als einer der 15 besten Beiträge für den „Best Paper Award“ nominiert. 


\section{Risikowahrnehmung in Milchviehbetrieben: Eine empirische Studie zur vergleichenden Bewertung von Politik-, Markt- und Produktionsrisiken}

Christian Wocken, Christian Schaper, Birthe Lassen, Achim Spiller und Ludwig Theuvsen

Dieser Beitrag wurde auf der 48. Jahrestagung der Gewisola zum Thema „Risiken in der Agrarund Ernährungswirtschaft und ihre Bewältigung " in Bonn vom 24.-26. September 2008 vorgestellt und wurde in den Schriften der Gesellschaft für Wirtschafts- und Sozialwissenschaften des Landbaus e.V., Band 44, 2009, Seiten 155-167 veröffentlicht. 


\section{Risk Management in Milk Production: A Study in Five European Countries}

Christian Schaper, Birthe Lassen und Ludwig Theuvsen

Dieser Beitrag wurde auf dem $113^{\text {th }}$ EAAE Seminar „A resilient European food industry and food chain in a challenging world", vom 03. bis 06. Septemper 2009 in Chania, Griechenland vorgestellt. Der Beitrag ist im Journal of Food Economics - Acta Agricult Scand C, 7, 2010, Seiten 5668, erschienen. 
5 Zusammenfassung und Ausblick/Summary 
Die zunehmende Liberalisierung der Gemeinsamen Agrarpolitik führt dazu, dass sich Milcherzeuger und Verarbeiter neu im überregionalen und internationalen Wettbewerb orientieren müssen. Durch das Ende der Milchquotenregelung kann es künftig zu Veränderungen der Marktanteile einzelner Regionen oder Länder auf dem Milchmarkt kommen. Gleichzeitig führt u. a. die Reduzierung von Interventionspreisen und Exporterstattungen dazu, dass sich Entwicklungen auf dem Weltmilchmarkt zunehmend auch in deutschen und europäischen Milchpreisen widerspiegeln und die Milcherzeuger somit neuen Marktund Preisrisiken gegenüberstehen. Aus diesen neuen Rahmenbedingungen entstehen zwei Forschungsfragen: (1) Welche regionalen Entwicklungen sind in der Milchproduktion künftig zu erwarten? (2) Wie passen sich Milcherzeuger strategisch und produktionstechnisch an die neuen Herausforderungen an?

Im Rahmen der vorliegenden Dissertation wurden die genannten Forschungsfragen unter gleichzeitiger Einbeziehung verschiedener etablierter Forschungsansätze (Produktionskosten-, Marktanteils-, Standortfaktorenanalyse) untersucht. Darüber hinaus wurde ein Forschungsansatz entwickelt, der es ermöglicht, international eine große Anzahl von Milcherzeugern einzubeziehen und so systematisch die Einschätzung der Hauptakteure im Markt zu erfassen (EDF-agri benchmark Snapshot). Dieser Ansatz wurde beispielhaft sowohl auf die Frage der regionalen Wanderung der Milchproduktion als auch auf die Frage künftiger produktionstechnischer Entwicklungen in der Melktechnik und ihrer Auswirkungen auf die Produktivitäten der Betriebe angewendet. Die in den vorliegenden publizierten (oder zur Publikation angenommenen) Beiträgen durchgeführten Analysen konzentrieren sich dabei auf Deutschland und ausgewählte Milcherzeugungsregionen in Europa.

\section{Künftige regionale Entwicklungen in der Milchproduktion}

Die Analyse künftiger regionaler Veränderungen in der Milchproduktion wurde zunächst auf Basis von drei unterschiedlichen analytischen Ansätzen vorgenommen: Marktanteils-, Produktionskosten- und Standortfaktorenanalyse. Da sich die Ergebnisse der unterschiedlichen Methoden teilweise widersprachen, wurden zwei weitere Ansätze im Rahmen der Dissertation entwickelt und ergänzend angewendet: (a) Standardisierte Expertengespräche in ausgewählten Regionen und (b) betriebsindividuelle Befragungen.

Die Analysen zeigen für Deutschland, dass die Milchproduktion in der Vergangenheit eher in Grünlandregionen gewandert ist. Gleichzeitig lässt sich ein stetiger Betriebsgrößenstrukturwandel erkennen, der künftig schneller erfolgen könnte als bisher. Dies könnte dazu führen, dass sich die Milchviehhaltung künftig verstärkt in Gemischtregionen konzentriert, da das Management größerer Herden in einigen Grünlandregionen aufgrund vermehrter Stallhaltung und erschwerter Mechanisierung der Flächen schwierig ist. Die Auswirkungen des Betriebsgrößenwachstums auf die regionale Verteilung der Milchproduktion innerhalb Europas ist schwer einzuschätzen, da nur wenige Länder mit besonders hohen (IE, UK, CH) oder besonders niedrigen (SE, HU, DK, BU, FL, ZY) Grünlandanteilen ausgestattet sind. 
In welchem Ausmaße sich eine Wanderung der Milchproduktion in Grünland- oder Gemischtregionen zeigen wird, hängt in erster Linie vom künftigen Preisniveau für Ackerfrüchte und von politischen Regelungen zum Grünlandumbruch ab. Darüber hinaus wird der künftige technische Fortschritt ebenfalls einen Einfluss auf die regionale Entwicklung der Milchproduktion haben, je nachdem, ob dadurch eher die Wettbewerbssituation größer oder kleiner strukturierter Betriebe verbessert wird.

Praktische Milcherzeuger in Deutschland und ausgewählten europäischen Milchregionen erwarten künftig ein beschleunigtes Herdengrößenwachstum. Sie gehen davon aus, dass die Milchproduktion im nördlichen und östlichen Zentraleuropa stärker ansteigen wird als in Südeuropa. Gleichzeitig sehen Betriebsleiter in viehintensiven Regionen Europas die knappe Landverfügbarkeit als Problem und erwarten deshalb begrenzte Expansionsmöglichkeiten für Milchviehbetriebe in diesen Regionen.

Obwohl die Aussagen der befragten Milcherzeuger nicht als repräsentativ für die Gesamtheit der europäischen Milcherzeuger zu sehen sind, stellen sie eine wertvolle Ergänzung zu den agrarstatistischen Analysen dar. Um künftig verlässlichere Aussagen zur regionalen Wanderung der Milchproduktion zu erzielen, sollten die Teilnehmerzahlen in den einzelnen Regionen soweit erhöht werden, dass eine nachträgliche Gewichtung der Daten auf Basis von Merkmalen der Grundgesamtheit möglich wird. Darüber hinaus sollte der intraregionale Strukturwandel ebenfalls berücksichtigt werden, beispielsweise durch Einschätzungen der Milcherzeuger zur regionalen Entwicklung der Milchproduktion. Eine Gewichtung der Einzeldaten und die Berücksichtigung von regionalen Einschätzungen konnten im Rahmen der vorliegenden Dissertation bereits für Niedersachsen angewendet werden. Um diese Vorgehensweise auch in anderen wichtigen Milcherzeugungsregionen anwenden zu können, ist jedoch die Ausweitung der Stichprobengröße notwendig.

Die einzelnen Analyseergebnisse erlauben eine besser abgestützte Einschätzung der künftigen regionalen Entwicklung der Milchproduktion. Eine abschließende Gesamtaussage kann jedoch nicht getroffen werden, da sich die Ergebnisse teilweise widersprechen und eine Gewichtung der einzelnen Methoden schwer möglich ist. Eine Überprüfung der Analyseergebnisse ist erst in einigen Jahren möglich, da durch die Veränderung der politischen Rahmenbedingungen derzeit keine Möglichkeit besteht, die Prognosefähigkeit der methodischen Ansätze auf Basis zurückliegender Entwicklungen zu überprüfen („Quasiex-ante-Analysen“). Dies ändert sich jedoch im Zeitverlauf und sofern es nicht erneut zu großen, politischen Umbrüchen kommt, ist es dann möglich, die Qualität der jeweiligen Prognosen rückwirkend zu bewerten und so auch eine Gewichtung der verschiedenen Analysemethoden vorzunehmen.

Erweisen sich dann die Ergebnisse als belastbare Prognoseindikatoren, wäre es sinnvoll, diese in internationale Marktmodelle einzubinden. Dies wäre wichtig, da die bisherigen Analysen nur die Angebotsseite umfassen, Veränderungen der Nachfrage jedoch nicht 
berücksichtigt Um zu einer umfassenden Einschätzung künftiger Entwicklungen auf dem Weltmilchmarkt zu gelangen, wäre jedoch die Rückkopplung zwischen Angebot und Nachfrage notwendig.

\section{Zusammenhang zwischen produktionstechnischen Entwicklungen und Produktivitä- ten der Betriebe}

Trotz mangelnder regionaler Repräsentativität kann das entwickelte Befragungskonzept heute schon genutzt werden, um Zusammenhänge zwischen Betriebsstrukturen, produktionstechnischen Entwicklungen und Produktivitäten der teilnehmenden Betriebe zu ermitteln. Da die Arbeitsproduktivität im Melkstand einen hohen Einfluss auf die Lohnkosten der Betriebe hat und damit besonders wichtig für eine rentable Milchproduktion ist, wurde der Befragungsansatz zunächst in diesem Bereich angewendet. Insgesamt 2.611 Milcherzeuger aus 19 Ländern Europas beteiligten sich an der Umfrage. Mittels Kontingenz- und Varianzanalysen konnten in der Stichprobe statistisch signifikante Zusammenhänge zwischen Betriebs- oder Standortfaktoren und der Wahl der Melktechnik sowie Einflüsse der Melktechnik auf die Arbeitsproduktivität der Betriebe festgestellt werden.

Zum Zeitpunkt der Befragung (Frühjahr 2011) wurden die meisten Kühe (42\%) in den befragten Betrieben in Fischgrätenmelkständen gemolken. Für die nächsten fünf Jahre (bis 2016) erwarten die befragten Milcherzeuger jedoch einen Rückgang der Fischgrätenmelkstände. Gleichzeitig gehen sie davon aus, dass künftig mehr Kühe in automatischen Melksystemen und Melkkarussellen gemolken werden. Rohrmelkanlagen und Tandemmelkstände verlieren bis 2016 in der Stichprobe nahezu vollständig an Bedeutung. Welches Melksystem in den Betrieben installiert wird, hängt signifikant von betrieblichen Aspekten (Herdengröße, Erwerbsform, Weidehaltung, Milchleistung), länderspezifischen Standortfaktoren (u. a. Lohnniveau) und von Persönlichkeitsmerkmalen der Betriebsleiter (Alter, Technikaffinität) ab. Einfluss auf die Produktivität der eingesetzten Melktechnologie haben bei automatischen Melksystemen vor allem die Besatzdichte und die durchschnittliche Milchleistung der Herde. Bei Melkkarussellen und Gruppenmelkständen wird die Arbeitsproduktivität hingegen von der Anzahl der Melker und dem Alter der Melktechnik (und damit auch der Ausstattung) beeinflusst.

Die erzielten Ergebnisse erlauben es Milcherzeugern, produktionstechnische Entwicklungen in anderen Ländern und deren Bedeutung für die eigene Wettbewerbsfähigkeit besser einzuschätzen. Gleichzeitig bleiben jedoch einige Fragen offen, die dem Befragungsdesign geschuldet sind: Da die Teilnehmer den Fragebogen freiwillig ausfüllen, muss dieser möglichst kurz sein. Die Abfrage von komplexen Sachverhalten oder Daten, die die Milcherzeuger nicht im Gedächtnis haben, ist somit nicht möglich. Neben einer Ausdehnung der Stichprobe im europäischen Ausland ist es deshalb künftig wichtig, die Befragungsdaten mit anderen vorliegenden Datensätzen verknüpfen zu können, beispielsweise mit dem EDF-Produktionskostenvergleich, um so detaillierte Analysen zum Einfluss produktions- 
technischer Entwicklungen auf das betriebswirtschaftliche Ergebnis der Betriebe zu ermöglichen.

\section{Risikowahrnehmung und Risikomanagement in Milchvieh haltenden Betrieben}

Eine zentrale Eigenschaft des EDF-agri benchmark Snapshots ist die Kürze des Fragebogens. Diese fördert einen hohen Rücklauf der Fragebögen. Daher eignet sich der Ansatz nicht für Themenstellungen, die umfangreichere Befragungen erfordern. Im Rahmen von kleineren Stichproben mit Fallstudiencharakter und ergänzenden Expertengesprächen können jedoch tiefergehende Analysen durchgeführt werden. Die verhaltenswissenschaftlichen Analysen zum Risikomanagement in Milchviehbetrieben in fünf europäischen Ländern (DE, FR, IE, NL, SE) wurden in Anbetracht der Komplexität des Themas deshalb in Form von Fallstudien durchgeführt und deren Ergebnisse im Rahmen von Expertengesprächen verifiziert. In den fünf Ländern beteiligten sich insgesamt 236 Milcherzeuger. Wie auch die Ergebnisse der EDF-agri benchmark Snapshot-Befragungen lässt die genutzte Stichprobe eher Aussagen für größere Milchviehbetriebe zu.

Die teilnehmenden Milchviehhalter in den fünf Ländern schätzen künftige Risiken weitgehend ähnlich ein: Marktrisiken messen sie tendenziell die höchste Bedeutung bei, gefolgt von Politik- und Produktionsrisiken. Die Gesamtbewertung der Einzelrisiken wurde unter Berücksichtigung der vermuteten Eintrittswahrscheinlichkeit und der erwarteten Schadensauswirkung vorgenommen. Insbesondere steigenden Preisen für Futtermittel und Pachtflächen wird ein hohes Risiko beigemessen, gefolgt von höheren (Umwelt-) Auflagen oder einer Verschärfung von Cross-Compliance-Richtlinien. Einzelne Risiken werden in den Ländern jedoch auch unterschiedlich wahrgenommen. So bewerten beispielsweise die französischen Teilnehmer die Schadensauswirkung einer Molkereiinsolvenz deutlich höher als die Teilnehmer in den anderen Ländern. Teilnehmer in Frankreich, der Schweiz und in Irland messen Tierseuchen eine höhere Bedeutung bei als Teilnehmer in den Niederlanden. Experten bestätigten die Einschätzungen der Milcherzeuger.

Die Ergebnisse zeigen, dass Milchviehhalter insgesamt risikobewusst agieren: Sie sind bereit, Risiken einzugehen, sofern betriebliche Belange dies erfordern. Diese Form der Risikoakzeptanz spiegelt sich auch insgesamt im Risikomanagement wider: Produktivitätssteigerung, Rationalisierung und Wachstum werden häufiger strategisch umgesetzt als Risikoverminderungsstrategien (beispielsweise Einkaufskooperationen für Inputs) oder Risikoüberwälzungsstrategien (z. B. Versicherungen). Zwar haben die Milcherzeuger für wichtige Schadensfälle Versicherungen, Risiken mit geringerer Schadensauswirkung werden jedoch eher nicht versichert. Lediglich in Frankreich entscheiden sich Milcherzeuger häufiger für Risikoverminderungsstrategien als für Wachstumsstrategien (z. B. in Form von Kooperationen). Dies ist auf die Regelungen des Milchquotenverkaufs zurückzuführen. 
Die Ergebnisse zeigen, dass viele Milcherzeuger neben den Risiken der Liberalisierung auch die Chancen eines freieren Milchmarktes erkennen. Deshalb ist es wichtig, dass ihnen künftig vermehrt durch internationale Analysen die Möglichkeit gegeben wird, strategische und produktionstechnische Entwicklungen im überregionalen und internationalen Umfeld rechtzeitig wahrzunehmen und deren Bedeutung für die eigene Wettbewerbsfähigkeit besser einzuschätzen.

\section{Summary}

The liberalisation of the Common Agricultural Policy (CAP) is forcing dairy farmers and dairy processors to face national and international competition. Changes in the milk market are to be expected due to the phasing out of the milk quota such that market shares of regions or countries might shift. At the same time, European and German milk prices more and more reflect the world market for milk because of the decrease in intervention prices and export subsidies. Dairy farmers, thus, face new market and price risks. Two research questions emerge regarding these new framework conditions: (1) What regional developments are to be expected in dairy production in the future? (2) How do dairy farmers adapt strategically and technologically to the new challenges?

In this dissertation the named research questions have been addressed using different established approaches at the same time (analyses of production costs, market shares and side factors). In addition, a new approach has been developed that allows surveying many dairy farmers on an international scale (EDF-agri benchmark Snapshot). Hence, it systematically captures the estimations of the main players in the market. This approach has been used for questions on future regional developments in dairy production as well as on questions about future technical developments in the field of milking technologies and their implications on the productivity of dairy farms. Analyses in the presented articles (all of which published or accepted for publication) focus on Germany and selected dairying regions in Europe.

\section{Future regional developments in dairy production}

Analyses of future regional trends in dairy production were first conducted with three different analytical approaches: analysis of market shares, production costs and side factors. Since results of the different approaches were partly contradictory, two more approaches were established in this dissertation and have been used in addition: (a) standardised interviews with experts in selected regions and (b) a farm survey.

Analyses for Germany show that in the past dairy production has increased in grassland areas. At the same time a continuous structural change towards larger herd sizes has been observed which, in the future, could proceed faster than in the past. This could move dairy production more into mixed areas, as the management of larger herds is more difficult in 
many grassland areas: Cows in larger herds usually graze less, but often grassland mechanisation is difficult. Implications of growing herd sizes within Europe are hard to estimate as there are only few countries with especially high (IE, UK, $\mathrm{CH}$ ) or especially low (SE, HU, DK BU, FL, ZY) shares of grassland.

The degree to which dairy production is shifting into grassland or mixed areas depends on the future price level for arable crops and on political regulations regarding the ploughing of grassland. At the same time technological progress will have an effect on regional developments in dairy production, when improving the competitiveness of either larger or smaller herds.

Active dairy farmers in Germany and selected European dairy regions expect accelerating herd size growth in future. They expect a stronger increase in dairy production in Northern and Eastern Europe than in Southern Europe. At the same time they regard land scarcity as a challenge in regions with high animal density and therefore expect fewer possibilities for expansion in those regions.

Even though conclusions of the surveyed dairy farmers cannot be used to make inferences for all European dairy farmers, they are adding valuable information to the analysis of agricultural statistics. In order to obtain more reliable information regarding the regional development of dairy production in the future, the number of survey participants should be increased. That allows ex-post-weighing of the sample according to indicators of the population. Also, the intraregional structural change should be considered, for example by asking for farmers' expectations for their regions. Both weighing of the sample and asking for regional estimations has been applied to a sample from Lower Saxony. In order to apply these approaches also to other important dairying regions, an enlarged sample size is necessary.

Results of the different analyses make the estimations on future regional developments in dairy production more robust. But an overall prediction still cannot be concluded, as results partly disagree with each other and it is not possible to assess the different approaches. A test of the results will only be possible a few years from now, since currently there are no possibilities to test the forecasting capability on the basis of past developments ('quasi ex ante analysis') due to the strong political changes. Given that policies stay rather stable in the near future, it will be possible to compare the forecasting capability of the different approaches by looking back. That will also allow a weighing of the different methods.

If results are accepted as valuable prognosis indicators, it would be interesting to combine them with international market models. This would be important because so far the analyses only examine the supply side of dairy production without looking at possible changes 
in demand. Yet, in order to obtain a comprehensive estimation of future developments on the milk market the interaction of supply and demand would be necessary.

\section{Relationship between production technology developments and productivity on dairy farms}

Even though the developed survey concept is not regionally representative, it can be used to detect relationships between farm structures, developments in production technologies and productivity of participating farms. As labour productivity in the milking parlour has a high impact on the labour costs of dairy farms and is therefore crucial for low production costs, the survey concept was first implemented in this context. In total 2,611 dairy farmers from 19 countries participated in the survey. Analysing data via contingency analysis and analysis of variance revealed statistically significant relationships between farm and side factors, and the used milking technology as well as impacts of different milking technologies on the labour productivity in the milking process.

At the time of the survey (spring 2011) the largest share of dairy cows (42\%) on the surveyed farms were milked in herringbone parlours. For the next five years (until 2016) participants expect a strong decrease in herring bones. At the same time they expect more cows to be milked in automatic milking systems and milking rotaries. The share of tandems and can milking systems in the sample is negligibly small for 2016 . The choice of a certain milking technology is influenced mainly by farm individual aspects (herd size, full time/part time farming, grazing, milk yield), country specific criteria (e.g., wages) and personal characteristics of the farm manager (age, technical affinity). The productivity of the different technologies is then influenced by different criteria: in automatic milking systems mainly the number of cows per box and the average milk yield influence productivity. In group milking parlours or rotaries productivity is mainly influenced by the number of milkers in the parlour and the age of the technology (also the equipment).

The results allow dairy farmers to better assess the developments in production technologies in other regions or countries as well as their implications for the own competitiveness. Still some questions remain open, mainly due to the survey design: As farmers fill in the questionnaire voluntarily, it has to be as short as possible. It is not possible to ask for complex details or figures that farmers do not have in mind at the time of the survey. Next to an enlargement of the sample within Europe it, therefore, is important to find a possibility to link the gained survey data to other existing data sets, e.g., EDF Cost of Comparison. That would allow a more detailed analysis about the influence of developments in production technologies on the economic result of the farms.

\section{Risk perception and risk management on dairy farms}

One key characteristic of the EDF-agri benchmark Snapshots is its briefness, which ensures a high return of questionnaires. However, the Snapshot is not suitable for topics that need more detailed questioning. But using smaller samples as case studies and adding ex- 
pert interviews allows in-depth analysis also in an international context. Behaviouristic analysis about risk management in dairy farms has been conducted in five different countries (DE, FR, IE, NL, SE) in the form of interviews and case studies because of the complexity of the topic. Results have been verified in expert interviews. A total of 236 dairy farmers participated in Germany, additional farmers joined in the five countries. Like the results of EDF-agri benchmark Snapshot, results represent the opinion of rather large dairy farms.

Participating dairy farmers in the different countries estimate future risks in a rather similar way: market risks were ranked highest, followed by political risks and production risks. Risk evaluation took into consideration both expected magnitude of the damage and perceived probability of the event. Especially increasing prices for feedstuff and increasing rent prices for land were estimated as high risks, followed by stricter (environmental) regulations or tighter Cross Compliance regulations. A few risks were evaluated differently between the countries. French farmers, for example, see a higher danger of an insolvency of their dairy processor than farmers in other countries. Farmers from France, Switzerland and Ireland estimate animal diseases as a higher risk than farmers from the Netherlands. Experts confirmed the evaluations of the farmers.

Results show that farmers are risk-conscious but are prepared to take risks if the farm management requires doing so. This risk acceptance is observable in most of the surveyed dairy farms: increasing productivity, rationalisation and growth are chosen over risk reduction strategies (e.g., co-operations for buying inputs) or risk transfer strategies (e.g., insurances). Dairy farmers do have insurance for risks with high damage potential but they often do not insure risks with lower damage potentials. Only in France risk reduction strategies are more common: farmers decide more often for co-operations than for growth strategies. This can be explained looking at specific regulations on quota transfer in France.

Analyses show that many dairy farmers also see chances - next to the risks of liberalisation - in a free dairy market. Hence, it is important for them to be able to judge international developments (strategically and technologically) and the implications for their own competitiveness. International analyses in this context are an important information tool for dairy farmers. 
6 Literaturverzeichnis 
Gömann H, Kreins P, Zabel A. (2006): Wohin wandert die Milchproduktion in Deutschland, Aktuelles zur Milcherzeugung, Landbauforschung SH 299, 97-108

Hollmann-Hespos T (2003): Risikomanagement im landwirtschaftlichen Betrieb - dargestellt anhand von Futterbaubetrieben in Niedersachsen. Masterarbeit Universität Göttingen

ISERMEYER F (2007): Zukunft der Milchquotenregelung - wie sind die verschiedenen Politikoptionen zu beurteilen? Braunschweig: FAL, Arbeitsberichte des Bereichs Agrarökonomie 2007/01

Isermeyer F, Brockmeier M, Gömann H, Hargens R, KLepper R, Kreins P, OfFermann F, Osterburg B, Pelikan J, Salamon P, Thiele H (2006): Analyse politischer Handlungsoptionen für den Milchmarkt. Landbauforschung SH 300

KÖHNE M (2000): LEITLINIEN FÜr BETRIEBSENTWICKLUNGEN. In: AgRARWIRTSCHAFT, 49. JG., H. 11, S.1-3

LUTTER M (2009): Strukturwandel in der europäischen Milchviehhaltung: Ergebnisse einer regional differenzierten Befragung, Masterarbeit an der Georg-AugustUniversität Göttingen

MiLChQuOTV (2008): Verordnung zur Durchführung der EU-Milchquotenregelung. URL: http://www.gesetze-im-internet.de/bundesrecht/milchquotv/gesamt.pdf, Abrufdatum: 24.03.2011

MILLER J (2005): Politische Rahmenbedingungen für die Milchviehhaltung in Bayern, in: Perspektiven in der Milchviehhaltung Schriftenreihe der Bayrischen Landesanstalt für Landwirtschaft

SCHAPER C et al. (2008): Risikomanagement in Milchviehbetrieben: Eine empirische Analyse vor dem Hintergrund der sich ändernden EU-Milchmarktpolitik In: Schriftenreihe der Landwirtschaftlichen Rentenbank. Band 23, Frankfurt 2008, S. 135-184 